\title{
Urinary Study on the Biochemical Effect of Acupuncture on Monosodium Urate Crystals-Induced Acute Gouty Arthritis in Rats using $600 \mathrm{MHz}{ }^{1} \mathrm{H}$ NMR
}

\author{
${ }^{1}$ West China Hospital, Sichuan University, Chengdu, 610041, PR China \\ ${ }^{2}$ Chengdu Women and Children Central Hospital, Chengdu, 610091, China \\ ${ }^{3}$ West China School of Pharmacy, Sichuan University, Chengdu, 610041, PR China \\ ${ }^{4}$ Analytical Center, Sichuan University, Chengdu, 610041, PR China \\ ${ }^{5}$ Laboratory Animal Center, Sichuan University, Chengdu, 610041, PR China
}

Yu Chen ${ }^{1}$, Li-Ping Wu², Yu-Jie Liu ${ }^{3}$, Peng-Chi Deng ${ }^{4}$, Hai-Lin Yin ${ }^{5}$, Si-Lan Wen ${ }^{3}$, Cong Chen ${ }^{3}$ and Li-Ming Ye ${ }^{3 *}$

\begin{abstract}
Acupuncture may potentially be an alternative therapy for acute gouty arthritis, a common inflammation model with multiple pathogenic mechanisms. In order to investigate the biological process of acute gouty arthritis and the effect of acupuncture, ${ }^{1} \mathrm{H}$ nuclear magnetic resonance (NMR)-based metabonomics has been applied to acquire urine metabolom data. Acute gouty arthritis model rats were induced by monosodium urate (MSU) crystals. The acupuncture on ST-36 (Zusanli) and SP-6 (Sanyinjiao) acupoints was applied for nine consecutive days. Principal Components Analysis (PCA) and Partial Least Squares (PLS) have been used to compare the time course urine metabolic perturbations. The prominent changes in endogenous urinary metabolites in acute gouty arthritis rats comprised increased levels of citrate and succinate, and decrease of allantoin, lactate, formate, trimethyl- $\mathrm{N}$-oxide (TMAO) and taurine on the $3^{\text {rd }}$ day after MSU injection; and on day 9, Citrate, succinate, and allantoin began to recover to the original level, whereas formate, taurine, TMAO and lactate further decreased, which showed their incompletely recovery. Acupuncture treatment appears to reverse the levels of most metabolites affected by acute gouty arthritis, however, with significant increase levels of acetate, acetoacetate, allantoin, creatine/creatinine, phenylacetylglycine (PAG), taurine and TMAO on day 3; all these metabolites began to recover to the original level on day 9 except for creatine/creatinine, which slightly increased, demonstrating the therapeutic effects of acupuncture on acute gouty arthritis. Our work shows the potential of an NMR-based metabonomic approach in the study of biological effects of acupuncture on acute gouty arthritis.
\end{abstract}

Keywords: ${ }^{1} \mathrm{H}$ NMR spectroscopy; Acupuncture; Acute gouty arthritis; Metabonomics; Multivariate analysis; Urinary metabolites

\section{Introduction}

Gouty arthritis is a common inflammation model with multiple pathogenic mechanisms seen in clinical practice, for which acupuncture is used as an alternative therapy. Previous UPLC-MS based metabonomic investigation proved that acupuncture therapy can relieve the metabolic perturbations during disease processes caused by MSU induced acute gouty arthritis; however, biomarkers had not been identified [1]. In order to search for the possible biomarkers, highthroughput analytical technique high resolution ${ }^{1} \mathrm{H}$ Nuclear magnetic resonance (NMR) and multivariate statistical analysis were applied in the present study.

Acupuncture, a non-pharmacological therapy in which thin needles are inserted into the body at specific spots called acupuncture points, has long been practiced for treating acute and chronic pain, and other diseases [2]. It's thought to activate receptors as well as nerve fibers located deep in the acupuncture points, cutaneous and deep tissues injury possibly accompany with subsequent local inflammatory responses and excitations of various nociceptors [3]. Generally, acupuncture influences a diverse set of body organs and functions including brain processing [4] and autonomic nervous system activity [5]. Secondly, it has long been known that the effects of acupuncture may actually continue long after needle stimulation [6]. Although many studies, like fMRI researches [7], have investigated brain response to acute needle stimulation, few have evaluated acupuncture effects on entire functional connectivity and in the period that extend beyond the active needle stimulation state, i.e. sustained acupuncture effects.
In the present study, we presume that metabonomics can be used to investigate the extended effect of acupuncture on acute gouty arthritis as well as to reveal its mechanism.

Metabonomics, defined as "the quantitative measurement of the dynamic multiparametric response of a living system to pathophysiological stimuli or genetic modification" [8], joins genomics, transcriptomics, and proteomic as a new significant branch of systems biology. The approach of metabonomics is able to investigate the physiological and pathologic changes of certain objects by measuring the complicated and dynamic variations of whole endogenous low molecular weight organic metabolites of cells, tissue and bio-fluids, other than quantify a limited set of the analytes contained in the metabolom. With multivariate analysis, the techniques of metabonomics provide a greater detail in biochemical process, which are particularly suitable for the research of living system's responses to exogenous stimulus. It

*Corresponding author: Li-Ming Ye, West China School of Pharmacy, Sichuan University, NO.17, Block 3, South Renmin Road, Chengdu, 610041, People's Republic of China, Tel: (+86) 13980986791; Fax: (+86) 028 85502305; E-mail: yeliminglaoshi@126.com

Received March 03, 2012; Accepted October 26, 2012; Published October 31, 2012

Citation: Chen Y, Wu LP, Liu YJ, Deng PC, Yin HL, et al. (2012) Urinary Study on the Biochemical Effect of Acupuncture on Monosodium Urate Crystals-Induced Acute Gouty Arthritis in Rats using 600MHz ${ }^{1} \mathrm{H}$ NMR. J Bioequiv Availab 4: $106-$ 111. doi:10.4172/jbb.1000122

Copyright: (c) 2012 Chen Y, et al. This is an open-access article distributed under the terms of the Creative Commons Attribution License, which permits unrestricted use, distribution, and reproduction in any medium, provided the original author and source are credited. 
has been shown to have enormous potential when applied to subjects as diverse plant genotype discrimination [9], toxicological mechanisms [10], disease processes [11,12], and drug discovery [13]. The process of acupuncture refers to entire biosystem, which properly coincides with the characteristic of metabonomics. Furthermore, metabonomics can reflect the dynamic changes of the body. Thus, metabonomics provides an effect way to investigate the process of acupuncture treatment on acute gouty arthritis. Besides, metabonomic technology will also contribute to the evaluation of the comprehensive effect, as well as the material basis and the safety of acupuncture, which will establish a scientific estimate method for treatment. However, to our knowledge, few papers focus on this field.

This investigation aims at confirming the effect of acupuncture therapy toward acute gouty arthritis through the analysis of metabolism pattern variation during therapy process, simultaneously, setting up the foundation of further searching for characteristic metabolites of acute gouty arthritis as well as revealing the mechanism of acupuncture therapy toward acute gouty arthritis.

\section{Materials and Methods}

\section{Experimental animals and model of acute gouty arthritis}

All animal procedures were performed according to the Guide for the Care and Use of Laboratory Animals of the National Institutes of Health, as well as the guidelines of the Animal Welfare Act. Adult male Sprague-Dawley (SD) rats weighing 180-210 g were obtained from Laboratory Animal Center, Sichuan University. Each animal was kept under controlled temperature $\left(20 \pm 2^{\circ} \mathrm{C}\right)$ and lighting conditions (08:00-20:00 h) with food and water made available ad libitum. The rats were randomly divided into three groups ( $n=5$ in each group): the acute gouty arthritis group (the model group); the acute gouty arthritis acupuncture-therapy group (the acupuncture group); the control group. Rat model of acute gouty arthritis was induced by MSU, which were synthesized by the method previously published [14], suspended in sterile saline and autoclaved for $30 \mathrm{~min}$ before use. $3.5 \mathrm{mg}$ MSU crystals suspended in $50 \mu \mathrm{l}$ of sterile saline was injected into unilateral ankle articular cavity of the hind limb of the rat in both the model group and the acupuncture group by a technique previously described in detail [15]. The control group received a $50 \mu \mathrm{l}$ intra-articular injection of single sterile saline.

\section{Acupuncture treatment and sample collection}

The stainless acupuncture needles of $0.3 \mathrm{~mm}$ diameter were inserted into the acupoints ST-36 and SP-6, and both left in place for $30 \mathrm{~min}$ as previously described in detail [1]. The acupuncture group was given acupunctural treatment for 9 consecutive days starting the first day after the establishment of acute gouty arthritis model. The rats freely acted in a piece of plat handled with special care to minimize stress. Urine samples of rats in each group were collected at certain time point as follows: before injection; 3 days and 9 days after MSU injection. Samples were immediately frozen after collection and stored at $-20^{\circ} \mathrm{C}$ before NMR analysis.

\section{Measurement of ankle perimeter}

To assess degree of inflammation, the circumference of the inflamed ankle joint of rats was measured using a $3 \mathrm{~mm}$ wide flexible paper without elasticity before and $2 \mathrm{~h}, 6 \mathrm{~h}, 10 \mathrm{~h}$ and each day until the end of experiment after MSU injection. To present amplitude of the reduction of edema, the data were converted to a percentage of changes of ankle size as a intumesce index using the following formula:
Intumesce Index $=($ measured ankle size - primary ankle size $) /$ primary ankle size

\section{${ }^{1} \mathrm{H}$ NMR spectroscopy analysis}

For NMR analysis, a $0.4-\mathrm{ml}$ sample of urine was mixed with phosphate buffer $\left(0.2 \mathrm{ml}, 0.2 \mathrm{M} \mathrm{Na}_{2} \mathrm{HPO}_{4} / 0.2 \mathrm{M} \mathrm{NaH}_{2} \mathrm{PO}_{4}\right.$ in $\mathrm{H}_{2} \mathrm{O}$, $\mathrm{pH} 7.1)$, and $\mathrm{D}_{2} \mathrm{O}(67 \mu \mathrm{l})$. The solutions were centrifuged at $14000 \mathrm{rmp}$ for $10 \mathrm{~min}$ at $4^{\circ} \mathrm{C}$. The supernatant was transferred to 5-mm NMR tubes to which a further 5 - $\mu$ l of $0.5 \mathrm{mM}$ TSP (sodium 3-trimethylsilyl[2,2,3,3-2 $\left.\mathrm{H}_{4}\right]$-1-propionate) in $\mathrm{D}_{2} \mathrm{O}$ was added. The buffer can lock nuclei and stabilizing the $\mathrm{pH}$ of the samples; the $\mathrm{D}_{2} \mathrm{O}$ provided a field frequency lock-solvent for the NMR spectrometer; and the TSP served as an internal chemical shift reference $(\delta 0.0 \mathrm{ppm})$.

All ${ }^{1} \mathrm{H}$ NMR experiments were carried out at $27^{\circ} \mathrm{C}$ on a Bruker AV II-600 MHz spectrometer by using the standard zgpr pulse sequence with water presaturation during the recycle delay of $5 \mathrm{~s}$ and a mixing time of $100 \mathrm{~ms}$. Each dataset was averaged over 32 transients using 32,000 time domain points.

All urine ${ }^{1} \mathrm{H}$ NMR spectra were manually phased and baseline corrected using MestreC (www.mestrec.com ), and then segmented into regions of $0.04 \mathrm{ppm}$. The area for each segmented region was calculated and the integral values contributed to an intensity distribution of the whole spectrum. The region $(\delta$ 4.65-5.0) was excluded prior to statistical analysis to remove the variation in water suppression efficiency; the region containing urea $(\delta$ 5.5-6.1) was excluded to eliminate any cross relaxation effects on the urea signal; and the regions containing TSP was also removed. All remaining regions of the spectra were then scaled to the total integrated area of the spectra to reduce any significant concentration differences. The bin size of 0.04 ppm typically used for ${ }^{1} \mathrm{H}$ NMR spectra is a compromise between the resolution and the variation in the position of resonance peaks from some metabolites, such as citrate, caused by $\mathrm{pH}$ variation, especially in urine [16]. The integrated data were normalized to the constant sum of integrals for each spectrum in order to reduce any significant concentration differences between samples, and then exported as text files for further multivariate statistical analysis.

\section{Data analysis}

The Intumesce Index data were expressed as means \pm SD and analyzed using the SPSS for windows, version 12.0 (SPSS Inc., Chicago, USA) statistical program. Statistical analyses were done using one-way analyses of variance (ANOVAs) followed by Student's Newman-Keul's test, $\mathrm{P}$ values less than 0.05 were considered significant.

The resulting integral data were imported into SIMCA-P (version 11.5; Umetrics, Umea, Sweden) for multivariate analysis, such as principal components analysis (PCA) and partial least squares (PLS). PCA is a non-supervised method [17], which was performed for data from different groups to detect the distributions and separations among groups; while PLS is a supervised method [18], which was used to find differential metabolites among groups. Prior to multivariate analysis, all data variables (bins) were mean-centered and Pareto scaled, i.e., dividing the (mean-centered) variables by the square root of their standard deviation. This scaling method reduces the larger variances more than the smaller ones, this effectively reduces the impact of noise [19]. The data was further preprocessed using orthogonal signal correction filter (OSC) to remove structured or systematic variation in responses from matrix $\mathrm{X}$ (dataset) that is not correlated or orthogonal 
to Y (group) [20], followed by PLS or PCA analysis. Two-dimensional Scores plots were used to visualize the separation of the groups, and the corresponding loadings plots reflect the contribution of each variable (NMR spectral regions) to the pattern in the scores plots.

In a model, a VIP (variable importance for the projection) values provided by SIMCA-P represent the ability to discriminate different classes of descriptors. In this study, a VIP>1.0 was set as the threshold for a meaningful contribution and compared with the results of univariate analysis to identify the significantly influential metabolites. The integrals of selected regions representing specific metabolites were subjected to two-tailed t-test. $\mathrm{p}<0.05$ was considered statistically significant.

\section{Results}

\section{Effects on ankle edema}

The ankle intumesce index induced by acute gouty arthritis were significant different among the 3 groups $(\mathrm{p}<0.05$, Figure 1 ). Measurement of the ankle index of the model group revealed an increase in ankle diameter than controls, while acupuncture treatment reduced the ankle diameter significantly in the acupuncture group.

\section{${ }^{1} \mathrm{H}$ NMR spectra of rat urine and the identification of urinary metabolites in ${ }^{1} \mathrm{H}$ NMR urinalysis}

${ }^{1} \mathrm{H}$ NMR analyses of rat urine highlighted a number of complex disturbances in the endogenous metabolites profiles, which could be related to both the acute gouty arthritis and acupuncture therapy induced biochemical pathway perturbation. Based on literature reports $[21,22]$, major metabolites in urine were identified, including amino acids, organic acids, lipids, and glucose (Figure 2).

\section{Principle component analysis in global profiling of urine samples and metabolic trajectory analysis}

In order to improve data analysis, data were pre-processed using orthogonal signal correction (OSC) filter with time as a correction factor. One component OSC was applied to the data sets before PCA and PLS analysis. To assess metabolic differences cause by acupuncture, ${ }^{1} \mathrm{H}$ NMR spectral urine profiles of rats were acquired and analyzed initially using PCA.

All urine samples collected from rats (three groups, $n=5$, three time

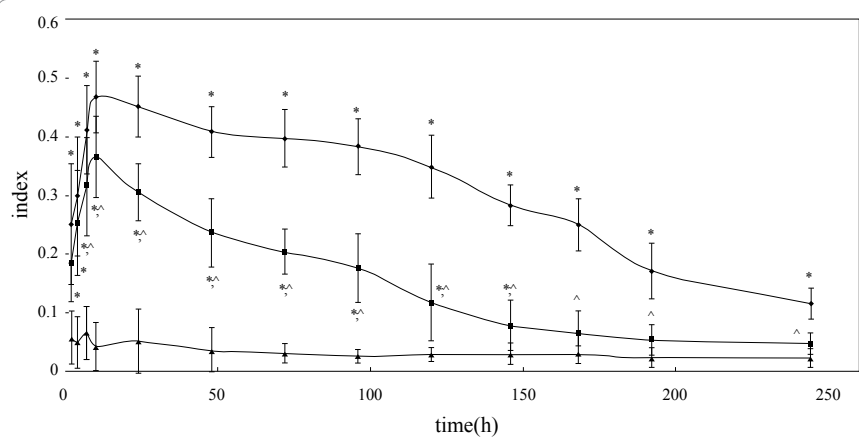

Figure 1: Effect of acupuncture on MSU crystal-induced ankle edema in rats. Group I: the model group; Group II: the acupuncture group; Group III: the control group. Values are expressed as mean $\pm \mathrm{SD}(n=5)$. Symbols * and ^ represent statistical significance at: $p<0.05$. Comparisons are made with a: ", represent Group I vs. Groups III, b: *, represent Group II vs. Groups III, c: ^’ represent Group II vs. Groups I. Statistical analysis was calculated by one way ANOVA followed by Student's Newman-Keul's test.

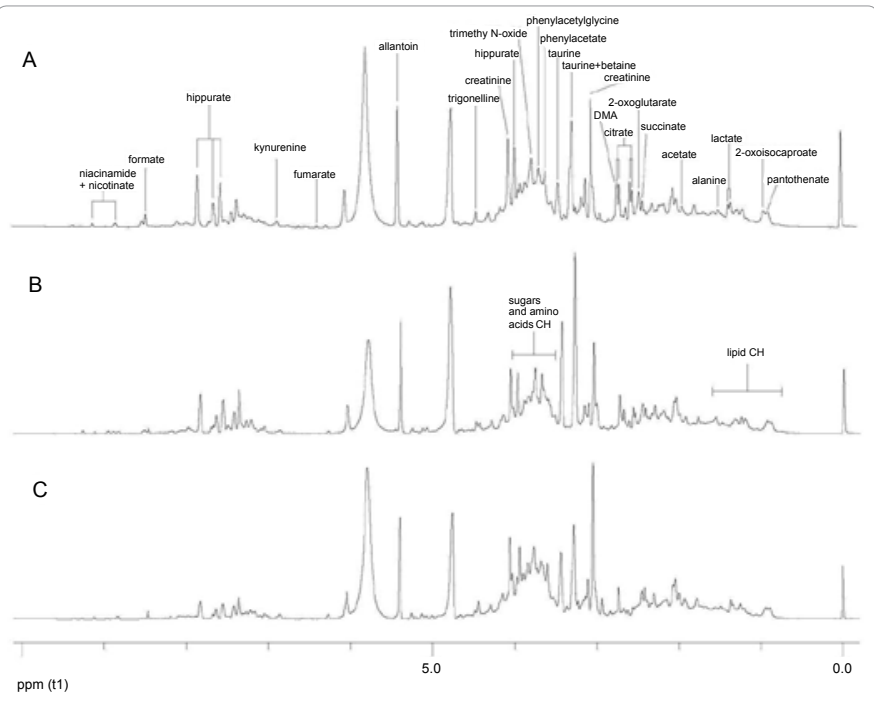

Figure 2: $600 \mathrm{MHz}{ }^{1} \mathrm{H}$ NMR spectra of urine samples collected 3 days after MSU administration from rats: $(A)$ the model group; (B) the acupuncture group; $(C)$ the control group (DMA, dimethylamine).

point) were applied to PCA analysis to investigate the time-dependent effect of MSU-induced acute gouty arthritis and acupuncture treatments over a 9-day experimental duration. The mean scores plots and loadings plots for the first two PCs at each time point were shown in (Figure 3).

This OSC-PCA method, contained 13 PCs $\left(\mathrm{R}^{2} \mathrm{X}(\mathrm{cum})=96.9 \%\right.$, $\left.\mathrm{Q}^{2}(\mathrm{cum})=79.5 \%\right)$, resulted in a marked separation in metabolic trajectory between the model group and the acupuncture group along PC1. The data were visualized by scores plots and loadings plots in (Figures 3A and 3B), respectively, where each point on the scores plot represented an individual urine sample and each point on the loadings plot represented a mass feature. Moreover, directions in the scores plot correspond to directions in the loadings plot.

The OSC-PCA metabolic trajectories of the urine profiles (Figure $3 \mathrm{~A}$ ) between the model and acupuncture groups according to collection time, except for individual data, showed that they moved away from the pre-dose position along PC1 axis on day 3 onwards. But they were different in that the model group further moved along PC2 with a maximum shift on day 9 while the acupuncture group started to return, however, the acupuncture group still different from pre-dose position along $\mathrm{PC} 1$ axis by the end of the monitoring period. There was no visible metabolic trajectory in the control group for the mean metabolic state of each time point clustered together near the origin which was not drawn in (Figure 3A). The prominent changes in endogenous urinary metabolites were confirmed by inspection of the OSC-PLS loadings plots and summarized in (Table 1).

PLS analysis in global profiling of urine samples from MSUinduced rats and the effects of acupuncture

PLS is a supervised analysis which finds directions in a multivariate space for maximum separation of observations (urine samples) belonging to Y (groups/time of administration). PLS was initially used to evaluate the model group, the acupuncture group and control group on the 3rd day, when difference among the three groups was distinguished. Then, PLS was used to inspect both the model group and the acupuncture group for the time depend metabolic trajectory. 
Citation: Chen Y, Wu LP, Liu YJ, Deng PC, Yin HL, et al. (2012) Urinary Study on the Biochemical Effect of Acupuncture on Monosodium Urate Crystals-Induced Acute Gouty Arthritis in Rats using 600MHz ${ }^{1}$ H NMR. J Bioequiv Availab 4: 106-111. doi:10.4172/jbb.1000122
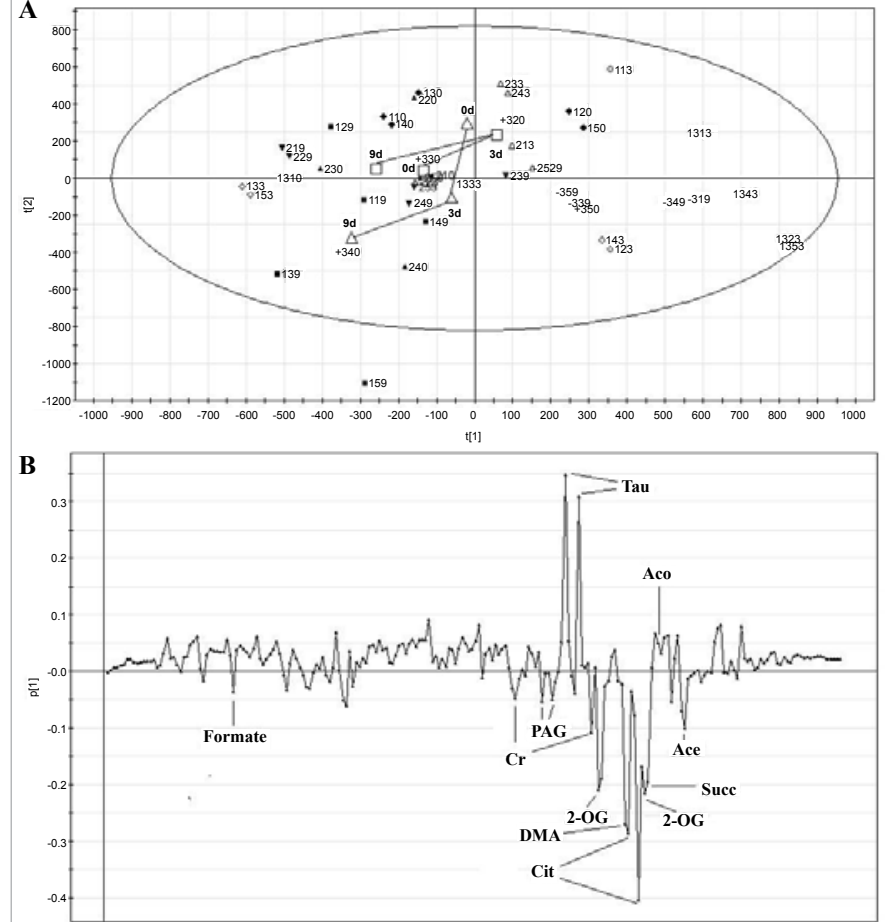

Figure 3: OSC-PCA scores (A) and loadings (B) plots based on ${ }^{1} \mathrm{H}$ NMR spectra of urine samples, before MSU injection (0d), 3 days after MSU injection (3d), and 9 days after MSU injection (9d), from the control group (0d, cross; $3 d$, vertical line; 9d, horizontal line; $n=5$ ) and the model group (0d, triangle; $3 d$, open triangle; $9 \mathrm{~d}$, inverted triangle; $\mathrm{n}=5$ ), and the acupuncture group (0d, diamond; $3 d$, open diamond; $9 d$, box; $n=5)$. First two PCs were calculated with explanation of $46.5 \%$ of total variances. Ellipses represent means $\pm S D$ for the various groups. $-\Delta-$, metabolic trajectory from the model group, $-\square-$, metabolic trajectory from the acupuncture group. Ace, acetate; Aco, acetoacetate; Cit, citrate; $\mathrm{Cr}$, creatine; Lac, lactate; 2-OG, O-acetylcarnitine; Succ, succinate; PAG, phenylacetylglycine; Tau, taurine.

\begin{tabular}{|c|c|c|c|c|c|}
\hline \multirow{2}{*}{ Metabolites } & \multirow{2}{*}{ Peak regions $(\delta)$} & \multicolumn{2}{|c|}{ arthritis } & \multicolumn{2}{|c|}{ acupuncture } \\
\hline & & $3 d$ & $9 d$ & $3 d$ & $9 d$ \\
\hline acetate & 1.92 & - & - & $\uparrow$ & - \\
\hline acetoacetate & 2.29 & - & - & $\uparrow$ & - \\
\hline allantoin & $5.83,6.03$ & $\downarrow$ & - & $\uparrow$ & - \\
\hline Lactate & 1.32 & $\downarrow$ & $\downarrow$ & - & - \\
\hline citrate & $2.69,2.55$ & $\uparrow$ & - & $\downarrow$ & - \\
\hline Succ & 2.42 & $\uparrow$ & - & - & - \\
\hline creatine and creatinine & $4.05,3.05$ & - & - & $\uparrow$ & $\uparrow$ \\
\hline PAG & $7.35,7.42,3.76,3.68$ & - & - & $\uparrow$ & - \\
\hline formate & 8.50 & $\downarrow$ & $\downarrow$ & - & - \\
\hline taurine & $3.27,3.42$ & $\downarrow$ & $\downarrow$ & $\uparrow$ & - \\
\hline TMAO & $3.30,3.26$ & $\downarrow$ & $\downarrow$ & $\uparrow$ & - \\
\hline
\end{tabular}

Note: $\uparrow$, indicates relative increase in signal; $\downarrow$, relative decrease in signal; -, no significant change according to the corresponding loadings plot. TMAO, trimethyl$\mathrm{N}$-oxide; PAG, phenylacetylglycine

Table 1: Changes of metabolites in the model and acupuncture groups relative to the control group.
Metabonomics evaluation of the 3rd day's evolution of rats' urine metabolites after injection of MSU

The OSC-PLS model of the 3rd day's rats' urine, contained two latent variables (LVs) characterized by $R^{2} X, R^{2} Y$ and $Q^{2}$ values of 0.796 , 1 and 0.998 , showed clear classification on urine metabolic profiles (Figure 4A), illustrated the metabolic distinction among the model group, the acupuncture group and the control group. The acupuncture group was visual separated and located clustering between the model group and control group in the LV1. Scores plot showed direct evidence reflecting the effect of acupuncture on acute gouty arthritis rats to relieve the metabolic changes. On the other hand, except three samples, there were differences between the acupuncture group and the other two groups along LV2 in scores plot (Figure 4A), which might be temporarily attributed to the needling effects of acupuncture treatment on the physiological condition. The prominent changes in endogenous urinary metabolites, confirmed by inspection of the loadings plot (Figure 4B) comprised increase in citrate and succinate, and decrease in lactate, taurine and TAMO in the model group than controls. While the urine levels of lactate and succinate in the acupuncture group were close to that before administration as compared to the model group, furthermore, urine levels of acetate, PAG, taurine and TMAO were increased, and the level of citrate was decreased in acupuncture group, which had reversed the changes in model group Based on the PLS loadings plot, a list of metabolites whose up-or-down regulation leads to the separation is presented in (Table 1).

Metabonomics evaluation of the model and acupuncture groups' evolution of rats' urine metabolites during experiment

OSC-PLS was applied to the investigation of the metabolic time
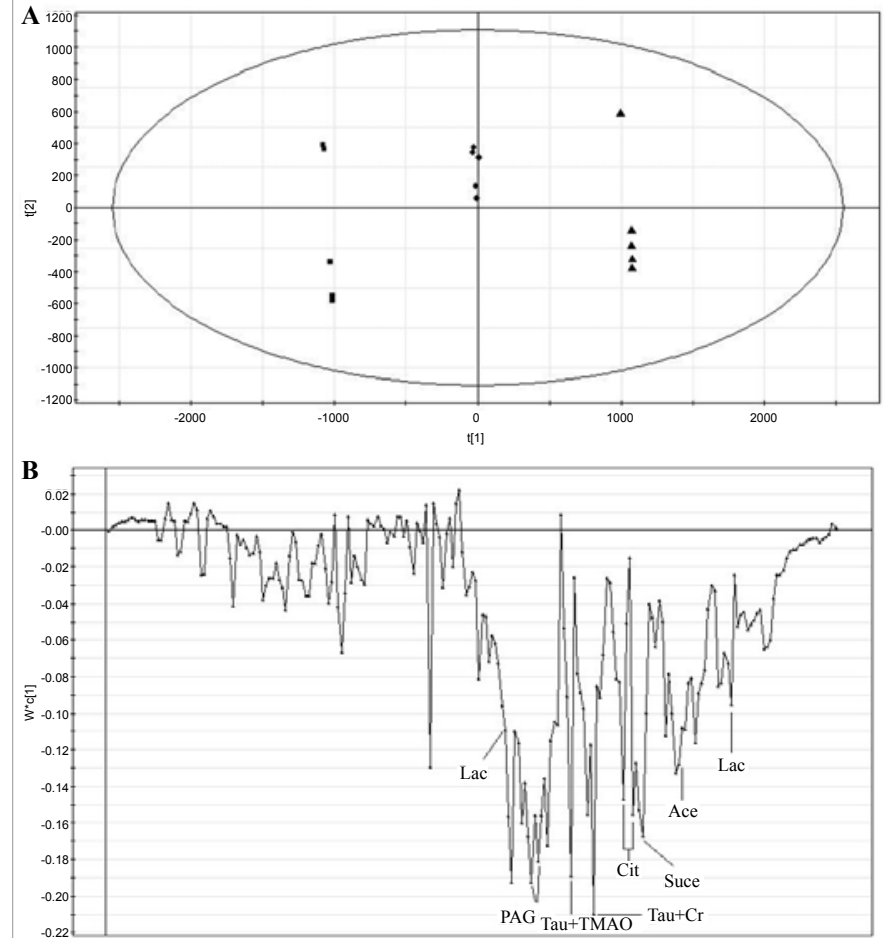

Figure 4: OSC-PLS scores (A) and loadings (B) plots based on ${ }^{1} \mathrm{H}$ NMR spectra of the $3^{\text {rd }}$ day's urine samples after injection of MSU from the control group ( $\mathbf{\square}$, $\mathrm{n}=5)$, the model group $(\boldsymbol{\Delta}, \mathrm{n}=5)$, and the acupuncture group $(\bullet, \mathrm{n}=5)$. (For $2 \mathrm{LVs}$ $\left.R^{2} X=0.796, R^{2} Y=1, Q^{2}=0.998\right)$. Ellipses represent means $\pm S D$ for the various groups. TMAO, trimethyl-N-oxide. 
Citation: Chen Y, Wu LP, Liu YJ, Deng PC, Yin HL, et al. (2012) Urinary Study on the Biochemical Effect of Acupuncture on Monosodium Urate Crystals-Induced Acute Gouty Arthritis in Rats using 600MHz ${ }^{1}$ H NMR. J Bioequiv Availab 4: 106-111. doi:10.4172/jbb.1000122

course events in the acute gouty arthritis and acupuncture-treated rats. The scores (LV1 vs. LV2) plots of urine ${ }^{1} \mathrm{H}$ NMR spectra from the model group and the acupuncture group at each time point were shown in (Figures 5A and 6A), respectively. The loadings plot showed the significant metabolic difference of the model group at each time point, (Figure 5B), whereas (Figure 6B) showed the significant metabolic difference of the acupuncture group at each time point. The OSC-PLS of the model group, contained three latent variables (LVs) characterized by $\mathrm{R}^{2} \mathrm{X}, \mathrm{R}^{2} \mathrm{Y}$ and $\mathrm{Q}^{2}$ values of $0.683,0.993$ and 0.975 , while the OSC-PLS of the acupuncture group contained two latent variables (LVs) characterized by $\mathrm{R}^{2} \mathrm{X}, \mathrm{R}^{2} \mathrm{Y}$ and $\mathrm{Q}^{2}$ values of $0.713,1$ and 0.997 .

Similar to PCA analysis, the separation among each time point in each group was remarkable. In the model group, it was found that the urine trajectories firstly moved away from the control position to day 3 positions along LV1 axis and then further moved to day 9 positions, which was far from original position. The metabolic trajectories from urine indicated inflammation effects for MSU. In the acupuncture group, day 9 position located between day 0 position and day 3 positions along LV1 axis. The metabolic trajectories from urine indicated an incomplete recovery for acupuncture therapy.

The metabolic perturbations during the monitoring period exhibited different tendencies between the model group and the acupuncture group according to the corresponding OSC-PLS loadings plots on LV1 (Figures 5B and 6B). In the model group, there is an increase urine level of citrate and succinate, and a decrease of formate, taurine, lactate, TMAO and allantoin on day 3 as compared with their normal state; on day 9, allantoin, citrate, and succinate began to recover to the original level, whereas the levels of formate, taurine, TMAO and lactate further decreased. Acupuncture treatment appears to reverse
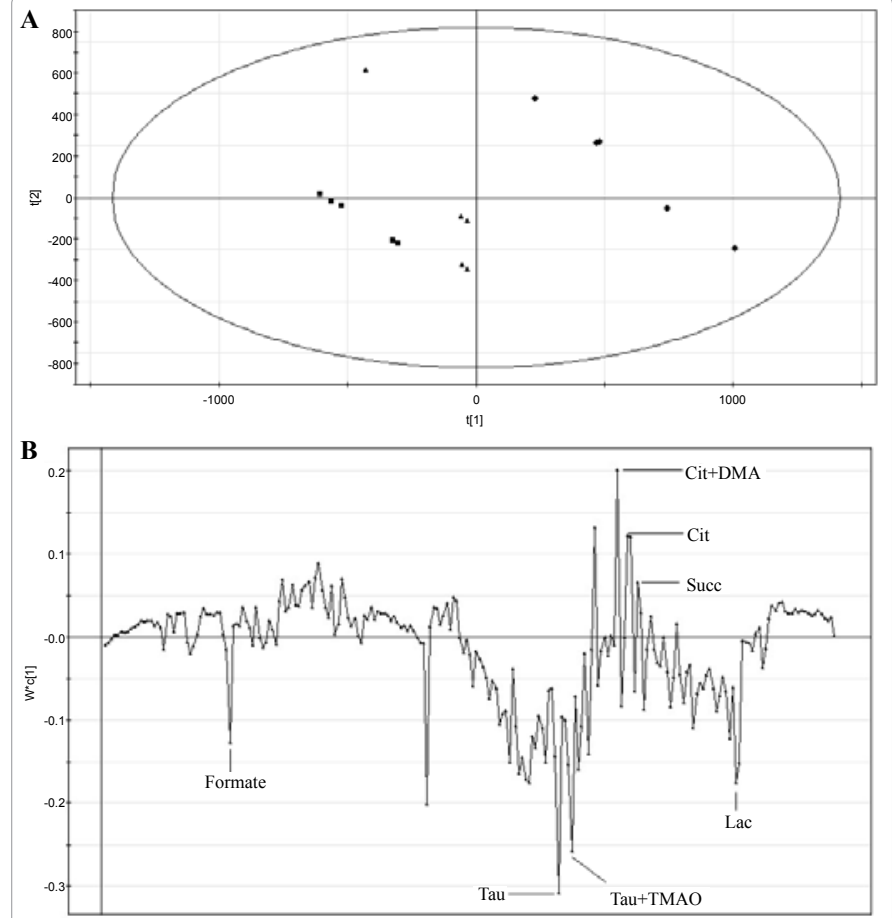

Figure 5: OSC-PLS scores (A) and loadings (B) plots from analysis of ${ }^{1} \mathrm{H}$ NMR spectra of urine samples from model group. $\mathbf{m}$, on day $0(n=5) ; \boldsymbol{\Lambda}$, on day 3 $(n=5)$; on day $9(n=5)$. (For first $\left.2 L V s, R^{2} X=0.504, R^{2} Y=0.971, Q^{2}=0.927\right)$. Ellipses represent means $\pm \mathrm{SD}$ for the various groups.
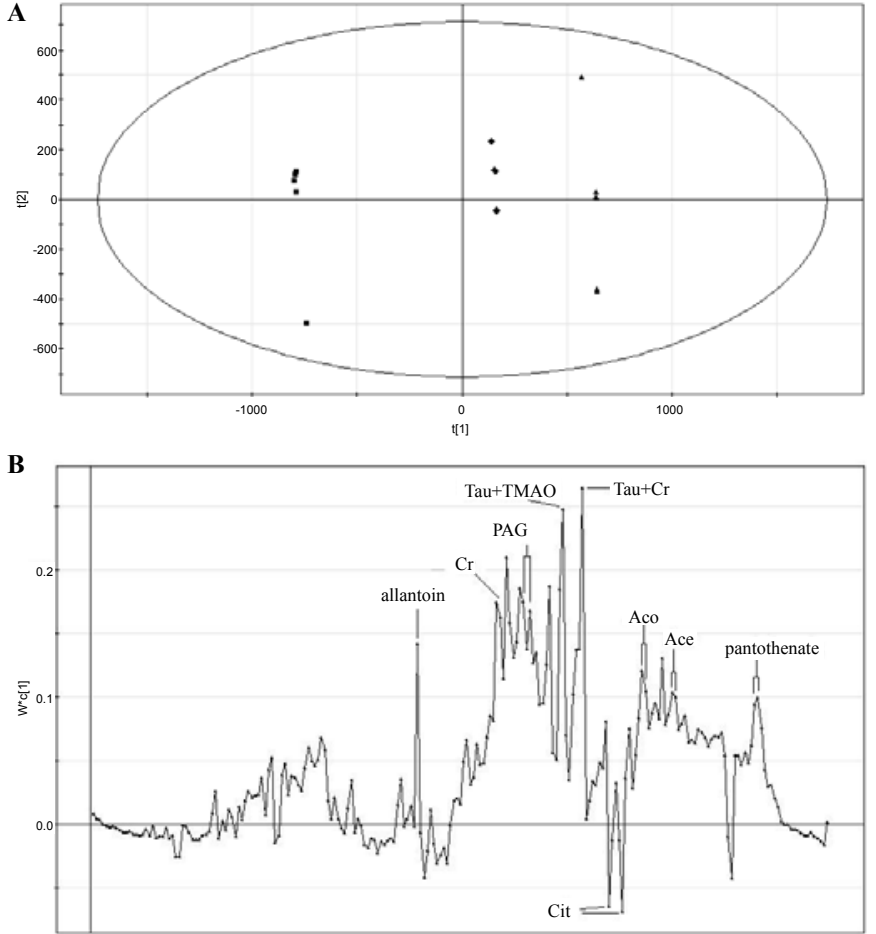

Figure 6: OSC-PLS scores (A) and loadings (B) plots from analysis of ${ }^{1} \mathrm{H}$ NMR

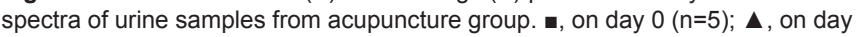
$3(n=5) ;$, on day $9(n=5)$. (For first $\left.2 L V s, R^{2} X=0.713, R^{2} Y=1, Q^{2}=0.997\right)$. Ellipses represent means \pm SD for the various groups.

the levels of most metabolites affected in acute gouty arthritis, however, with significant increase in the levels of allantoin, creatine/creatinine, taurine, TMAO, PAG, acetate and acetoacetate on day 3. All these metabolites began to recover to the original level, whereas creatine/ creatinine slightly increased on day 9. These metabolic perturbations are summarized in (Table 1).

\section{Discussion}

MSU-induced acute gouty arthritis inflammation model was triggered by a preformed deposit of crystals or by acute crystallization. MSU caused a brief inflammatory response, characterized by phagocytosis of particles and release of lysosomal enzymes by leucocytes in the production of inflammation, resulting in membranolysis $[23,24]$. This disease is characterized by the crystal deposition in the joint with marked influx of polymorphoneutophils, inflammatory edema and severe pain. Arthritis can cause disorder of sensorimotor function principally affecting quality of life. Therefore, the development of therapy for arthritic pain is of the utmost importance.

${ }^{1} \mathrm{H}$ NMR spectroscopy, as one of the most powerful tools for profiling of biofluids spectroscopy, is useful for measuring concentration with good reproducibility and nondiscriminatory detection. Using ${ }^{1} \mathrm{H}$ NMR spectroscopy to follow various biochemical responses does not require the pre-selection of metabolites and allows subsequent multicomponent analysis, without the bias imposed by the experimenters' expectations. Therefore, the NMR spectra of biosamples provide important information on endogenous biochemical processes in both healthy and disease states.

In this study, ${ }^{1} \mathrm{H}$ NMR-based metabonomics was used to 
Citation: Chen Y, Wu LP, Liu YJ, Deng PC, Yin HL, et al. (2012) Urinary Study on the Biochemical Effect of Acupuncture on Monosodium Urate Crystals-Induced Acute Gouty Arthritis in Rats using 600MHz ${ }^{1}$ H NMR. J Bioequiv Availab 4: 106-111. doi:10.4172/jbb.1000122

demonstrate the metabolic perturbation induced by experimental acute gouty arthritis and traditional acupuncture treatment. Several putative biomarkers for acute gouty arthritis and acupuncture therapy are found using ${ }^{1} \mathrm{H}$ NMR quantification and statistical analyses. Urine metabolic profiles showed that the maximum metabolic changes occurred three days after the injection of MSU. In acute gouty arthritis rats, there is an increase urine level of citrate and succinate, and a decrease of formate, taurine, lactate, TMAO and allantoin on day 3 as compared with their normal state; on day 9, citrate, succinate, and allantoin began to recover to the original level, whereas the levels of formate, taurine, TMAO and lactate further decreased. Acupuncture treatment appears to reverse the levels of most metabolites affected in acute gouty arthritis, however, with significant increase in the levels of allantoin, creatine/creatinine, taurine, TMAO, PAG, acetate and acetoacetate on day 3. All these metabolites began to recover to the original level, whereas creatine/ creatinine slightly increased on day 9 . The results show that energy metabolism and amino acid metabolism could be affected by acute gouty arthritis and acupuncture treatment. Acute gouty arthritis and acupuncture treatment presented slight kidney injury, which might be associated with an indirectly mechanism involving stress-induced oxidative stress, resulting in possible perturbation in the renal tubular function. NMR-based metabonomics has been proved to be an efficient technique to investigate the biochemical effects of acupuncture.

Our results suggest that the rats of acupuncture group probably recovered toward normal metabolism status earlier than the rats of model group due to certain relief function of acupuncture therapy toward acute gouty arthritis during the monitoring period. Acupuncture suppress edema associated with acute gouty arthritis and may have a beneficial role in the treatment of inflammatory, although further studies are needed to refine the predictive potential of these metabolites for acupuncture development as well as to determine the precise mechanism of action of acupuncture. We hypothesize that acupuncture was acting like the non-steroidal anti-inflammatory (NASID) agents by inhibiting the cyclooxygenase (COX)-mediated synthesis of prostglandins. A trial with more subjects and more detailed investigation is being planned, in which the mechanism of acupuncture therapy is compared to traditional NASID treatment. Moreover, additional research is needed to confirm these biomarkers because the other effects can be induced simultaneously during treatment. This established ${ }^{1} \mathrm{H}$ NMR technique-based metabonomics approach can provide a systematic and holistic view of the biochemical effect of nonpharmacological therapy as well as a simple and noninvasive method to investigate the effect of acupuncture on acute gouty arthritis in the future.

\section{Acknowledgements}

We gratefully acknowledge Professor Huang Jing from Sichuan University, Chengdu, Sichuan, P.R. China, for providing the SIMCA-P software and helpful discussions.

\section{References}

1. Wen SL, Liu YJ, Yin HL, Zhang L, Xiao J, et al. (2011) Effect of Acupuncture on Rats with Acute Gouty Arthritis Inflammation: A Metabonomic Method for Profiling of both Urine and Plasma Metabolic Perturbation. Am J Chin Med 39: 287-300.

2. Chernyak GV, Sessler DI (2005) Perioperative Acupuncture and Related Techniques. Anesthesiology 102: 1031-1049.

3. Yan Q, Ruan JW, Ding Y, Li WJ, Li Y, et al. (2011) Electro-acupuncture promotes differentiation of mesenchymal stem cells, regeneration of nerve fibers and partial functional recovery after spinal cord injury. Exp Toxicol Pathol 63: 151-156.
4. Dhond RP, Kettner N, Napadow V (2007) Neuroimaging acupuncture effects in the human brain. J Altern Complement Med 13: 603-616.

5. Han JS (2004) Acupuncture and endorphins. Neurosci Lett 361: 258-261.

6. Andersson S, Lundeberg T (1995) Acupuncture-from empiricism to science: functional background to acupuncture effects in pain and disease. Med Hypotheses 45: 271-281.

7. De Luca M, Beckmann CF, De Stefano N, Matthewsa PM, Smith SM (2006) $\mathrm{fMRI}$ resting state networks define distinct modes of long distance interactions in the human brain. Neuroimage 29: 1359-1367.

8. Raamsdonk LM, Teusink B, Broadhurst D, Zhang N, Hayes A, et al. (2001) A functional genomics strategy that uses metabolome data to reveal the phenotype of silent mutations. Nat Biotechnol 19: 45-50.

9. Fiehn $\mathrm{O}$ (2002) Metabolomics--the link between genotypes and phenotypes. Plant Mol Biol 48: 155-171

10. Nicholson JK, Connelly J, Lindon JC, Holmes E (2002) Metabonomics: a platform for studying drug toxicity and gene function. Nat Rev Drug Discov 1: 153-161.

11. Brindle JT, Antti H, Holmes E, Tranter G, Nicholson JK, et al. (2002) Rapid and noninvasive diagnosis of the presence and severity of coronary heart disease using 1H NMR-based metabonomics. Nat Med 8: 1439-1444.

12. Hasegawa M, Takenak S, Kuwamura M, Yamate J, Tsuyama S (2007) Urinary metabolic fingerprinting for amiodarone-induced phospholipidosis in rats using FT-ICR MS. Exp Toxicol Pathol 59: 115-120.

13. Moyec LL, Valensi P, Charniot JC, Hantz E, Albertini JP (2005) Serum $1 \mathrm{H}$-nuclear magnetic spectroscopy followed by principal component analysis and hierarchical cluster analysis to demonstrate effects of statins on hyperlipidemic patients. NMR biomed 18: 421-429.

14. Rasool M, Varalakshmi P (2006) Suppressive effect of Withania somnifera root powder on experimental gouty arthritis: An in vivo and in vitro study. Chem-Biol Interact 164: 174-180.

15. Sun S, Cao H, Han M, Li TT, Zhao ZQ, et al. (2008) Evidence for suppression of electroacupuncture on spinal glial activation and behavioral hypersensitivity in a rat model of monoarthritis. Brain Res Bull 75: 83-93.

16. Craig A, Cloarec O, Holmes E, Nicholson JK, Lindon JC (2006) Scaling and normalization effects in NMR spectroscopic metabonomic data sets. Anal Chem 78: 2262-2267.

17. Li L, Sun B, Zhang Q, Fang J, Ma K, et al. (2008) Metabonomic study on the toxicity of Hei-Shun-Pian, the processed lateral root of Aconitum carmichaelii Debx. (Ranunculaceae). J Ethnopharmacol 116: 561-568.

18. Antti H, Ebbels TMD, Keun HC, Bollard ME, Beckonert O, et al. (2004) Statistical experimental design and partial least squares regression analysis of biofluid metabonomic NMR and clinical chemistry data for screening of adverse drug effects. Chemometr Intell Lab 73: 139-149.

19. Jones GL, Sang E, Goddard C, Mortishire-Smith RJ, Sweatman BC, et al (2005) A functional analysis of mouse models of cardiac disease through metabolic profiling. J Biol Chem 280: 7530-7539.

20. Weljie AM, Dowlatabadi R, Miller BJ, Vogel HJ, Jirik FR (2007) An Inflammatory Arthritis-Associated Metabolite Biomarker Pattern Revealed by ${ }^{1} \mathrm{H}$ NMR Spectroscopy. J Proteome Res 6: 3456-3464.

21. Lindon JC, Nicholson JK, Holmes E (2007) Handbook of Metabonomics and Metabolomics. Elsevier, Oxford.

22. Nicholson JK, Foxall PJD, Spraul M, Farrant RD, Lindon JC (1995) 750 MHz $1 \mathrm{H}$ and $1 \mathrm{H}-13 \mathrm{C}$ NMR spectroscopy of human blood plasma. Anal Chem 67 : 793-811.

23. Glatt M, Dieppe P, Willoughby D (1979) Crystal-induced inflammation, enzyme release and the effects of drugs in the rat pleural space. J Rheumatol 6: 251258.

24. Woolf AD, Dieppe PA (1987) Mediators of crystal-induced inflammation in the joint. Brit Med Bull 43: 429-444. 